\title{
ORIGINAL ARTICLE Neuroimaging evidence of brain abnormalities in mastocytosis
}

\author{
N Boddaert ${ }^{1,16}$, A Salvador ${ }^{2,3,16}$, MO Chandesris ${ }^{4,5}$, H Lemaître ${ }^{1}$, D Grévent ${ }^{1}$, C Gauthier ${ }^{3}$, O Naggara ${ }^{6}$, S Georgin-Lavialle ${ }^{4,7,8}$, \\ DS Moura $^{4,7,9}$, F Munsch $^{10,11}$, N Jaafari ${ }^{12}$, M Zilbovicius ${ }^{1}$, O Lortholary ${ }^{4,13,14}$, R Gaillard $^{2,3,4,15,17}$ and O Hermine ${ }^{4,5,7,17}$
}

Mastocytosis is a rare disease in which chronic symptoms are related to mast cell accumulation and activation. Patients can display depression-anxiety-like symptoms and cognitive impairment. The pathophysiology of these symptoms may be associated with tissular mast cell infiltration, mast cell mediator release or both. The objective of this study is to perform morphological or functional brain analyses in mastocytosis to identify brain changes associated with this mast cell disorder. We performed a prospective and monocentric comparative study to evaluate the link between subjective psycho-cognitive complaints, psychiatric evaluation and objective medical data using magnetic resonance imaging with morphological and perfusion sequences (arterial spin-labeled perfusion) in 39 patients with mastocytosis compared with 33 healthy controls. In the test cohort of 39 mastocytosis patients with psycho-cognitive complaints, we found that $49 \%$ of them had morphological brain abnormalities, mainly abnormal punctuated white matter abnormalities (WMA). WMA were equally frequent in cutaneous mastocytosis patients and indolent forms of systemic mastocytosis patients ( $42 \%$ and $41 \%$ of patients with WMA, respectively). Patients with WMA showed increased perfusion in the putamen compared with patients without WMA and with healthy controls. Putamen perfusion was also negatively correlated with depression subscores. This study demonstrates, for we believe the first time, a high prevalence of morphological and functional abnormalities in the brains of mastocytosis patients with neuropsychiatric complaints. Further studies are required to determine the mechanism underpinning this association and to ascertain its specificity.

Translational Psychiatry (2017) 7, e1197; doi:10.1038/tp.2017.137; published online 8 August 2017

\section{INTRODUCTION}

Mastocytosis is a clonal disorder characterized by the accumulation of mast cells in one or more organs as well as their unregulated activation. ${ }^{1,2}$ It has been two decades since c-KIT mutations, mainly the D816V mutation, leading to constitutive activation of the C-KIT tyrosine kinase membrane receptor, were recognized as being central to the pathophysiology of mastocytosis. $^{3}$

The World Health Organization (WHO) classification of mastocytosis is regularly updated as well as the investigational recommendations for the diagnosis. ${ }^{4-7}$ Apart from the WHO classification $^{5,6}$ that recognizes seven types of mastocytosis, it is very important to distinguish between the life-threatening, aggressive forms of mastocytosis (aggressive systemic mastocytosis and mast cell leukemia with or without clonal hematological non-mast cell lineage disease and mast cell sarcoma) that involve organ damage with severe dysfunction (C-Findings), from other, more prevalent, indolent forms of mastocytosis. The latter include cutaneous mastocytosis (CM), indolent systemic mastocytosis (ISM) and smoldering systemic mastocytosis (SSM). These forms are characterized by a variable degree of mast cell accumulation in various organs (mainly the skin, bone marrow, and bowel) but without organ dysfunction (no C-Findings), and by a great variety of systemic symptoms resulting from mast cell mediator release (degranulation) secondary to autonomous and unregulated mast cell activation (MCA).

In 2008, we first reported an evaluation of patients' perceptions of disability in mastocytosis, stressing the under-evaluated burden caused by MCA. ${ }^{8}$ Because CM and ISM are by far the most prevalent, it is of major interest to better understand the mechanisms and heterogeneity of clinical expression associated with MCA to improve patient care. The mast cell mediator release symptoms are highly heterogeneous in type and intensity among patients and even in the same patient depending on time,

\footnotetext{
${ }^{1}$ Department of Pediatric Radiology, Hôpital Necker-Enfants Malades, AP-HP, University René Descartes, PRES Sorbonne Paris Cité, INSERM U1000 and UMR 1163, Institut Imagine, Paris, France; ${ }^{2}$ Laboratoire de "Physiopathologie des Maladies Psychiatriques", Centre de Psychiatrie et Neurosciences U894, INSERM, Université Paris Descartes, Sorbonne Paris Cité, Paris, France; ${ }^{3}$ Service de Psychiatrie, Centre Hospitalier Sainte-Anne, Université Paris Descartes, Sorbonne Paris Cité, Faculté de Médecine Paris Descartes, Paris, France; ${ }^{4}$ Centre de Référence des Mastocytoses, Université Paris Descartes, Sorbonne, Paris Cité, Hôpital Necker-Enfants Malades, Fondation Imagine, Paris, France; ${ }^{5}$ Service d'Hématologie Adulte, Université Paris Descartes, Sorbonne, Paris Cité, Assistance Publique-Hôpitaux de Paris, Institut Imagine, Hôpital Necker-Enfants Malades, Paris, France; ${ }^{6}$ Department of Neuroradiology, Service Hospitalo-Universitaire, Centre Hospitalier Sainte-Anne, Université Paris Descartes, Sorbonne Paris Cité, INSERM UMR894, Paris, France; ${ }^{7}$ INSERM U1163 and CNRS ERL 8254 and Laboratory of Physiopathology and Treatment of Hematological Disorders, Hôpital Necker-Enfants Malades, Paris, France; ${ }^{8}$ Service de Médecine Interne, Hôpital Tenon, Université Pierre et Marie Curie, Assistance Publique-Hôpitaux de Paris, Paris, France; ${ }^{9}$ Laboratoire de Psychopathologie et Processus de Santé, EA 4057, IUPDP, Institut de Psychologie, Université Paris Descartes, Paris, France; ${ }^{10}$ MRI France GE Healthcare, Vélizy Villacoublay, France and Université de Bordeaux, Bordeaux, France; ${ }^{11}$ Inserm U1215 - Neurocentre Magendie, Bordeaux, France; ${ }^{12}$ Unité de Recherche Clinique Intersectorielle en Psychiatrie à Vocation Régionale Pierre Deniker du Centre Hospitalier Henri Laborit, INSERM CIC-P 1402, INSERM U 1084 Laboratoire Expérimental et Clinique en Neurosciences, Univ Poitiers, CHU Poitiers, Groupement De Recherche CNRS 3557, Poitiers, France; ${ }^{13}$ Infectious Diseases Unit, Necker-Enfants Malades Hospital, AP-HP Paris, Paris, France; ${ }^{14}$ Paris Descartes University, Imagine Institute, Paris, France and ${ }^{15}$ Human Histopathology and Animal Models, Infection and Epidemiology Department, Institut Pasteur, Paris, France. Correspondence: Professor N Boddaert, MD, PhD, Department of Pediatric Radiology, Hôpital Necker-Enfants Malades, AP-HP, University René Descartes, PRES Sorbonne Paris Cité, INSERM U1000 and UMR 1163, Institut Imagine, 149 rue de Sèvres, 75015 Paris, France.
}

E-mail: nathalie.boddaert@nck.aphp.fr

${ }^{16}$ These authors contributed equally to this work.

${ }^{17}$ These authors contributed equally to this work

Received 21 December 2016; revised 6 April 2017; accepted 20 April 2017 
environment and treatment. Although some symptoms are strongly suggestive of mast cell activation (flush, anaphylactic reaction, and drug or food intolerances), most, if not all, mast cell mediator release symptoms are unspecific (for example, fatigue, musculoskeletal pains, gastrointestinal complaints, palpitations and malaise).

A large panel of neurological and psychiatric symptoms is also frequently reported by patients with mastocytosis including headaches, neuropathic pains, dizziness, attention and memory changes, anxiety, emotional over-reactivity, depressive-like symptoms and sleeping disorders. ${ }^{8-13}$ In a retrospective series of adult patients with a diagnosis of mastocytosis who were referred to a neurologist, multiple sclerosis has been found to be overrepresented. ${ }^{10}$ We have shown that 40 to $60 \%$ of mastocytosis patients present with psycho-cognitive manifestations including attention and memory impairments and depression. $8,11,12$ Although the number of publications on this topic is extremely scarce and the exact prevalence and specific characteristics are still not precisely validated, these symptoms tend to be considered authentic mast cell mediator release symptoms and should be carefully considered in medical coverage.

The present prospective comparative and monocentric study aimed to investigate relationships between psycho-cognitive complaints assessed via systematic psychiatric evaluation and objective medical data using magnetic resonance imaging (MRI) with morphological and perfusion sequences (arterial spin-labeled perfusion). In other systemic diseases such as systemic lupus erythematosus with neuropsychiatric symptoms (for a recent review, see Mikdashi, 2016) or even without (Mak et al., 2012), Sjögren's syndrome ${ }^{14}$ or Behçet's disease, ${ }^{15}$ MRI helped to characterize brain alterations related to psychiatric, cognitive or neurological symptoms. ${ }^{14-17}$ In mastocytosis, coupled psychiatric evaluation and MRI could not only demonstrate brain lesions and their correlates but could also pinpoint the involvement of mast cells in cognitive and depressive symptoms in a broader perspective.

\section{MATERIALS AND METHODS}

\section{Subjects}

Patients registered in the French National Reference Centre for Mast-cell diseases (centre de référence des mastocytoses, CEREMAST) were proposed to be enrolled in a French, prospective, monocentric clinical study. No formal sample size calculation was performed because mastocytosis is a rare condition and no preliminary data have allowed defining the size or even the type of neuroimaging results. Consecutive patients were recruited during 18 months at Necker Children's Hospital (Paris, France). Inclusion criteria included a diagnosis of a CM, ISM or SSM type of mastocytosis and the existence of psycho-cognitive symptoms/ complaints. The diagnosis of mastocytosis and its subtype was made by trained specialized medical doctors and characterized as either CM, ISM or SSM based on the WHO classification criteria., ${ }^{5,6}$ Systemic involvement relied on a bone marrow biopsy and/or aspirate with the presence of atypical mast cells expressing the immunostaining CD2 and/or CD25 as well as the presence of a D816V mutation in the C-KIT gene (see Supplementary Table). A diagnosis of CM was confirmed when either bone marrow analysis and blood tests indicated the absence of systemic involvement (no major criteria and less than three minor criteria, five patients) or when bone marrow analysis was not available (due to procedure failure or patient refusal, seven patients). Psycho-cognitive symptoms/complaints consisted of recurrent or continuous anxiety, depression or cognitive impairment, with consecutive feelings of dissatisfaction and/or disability in their current life.

Exclusion criteria included the presence of a known neurological disease such as epilepsy, multiple sclerosis or any identified comorbid systemic condition.

A control group including healthy volunteers free of mastocytosis as well as of any neurological and/or psychiatric disorders was constituted to perform brain imaging.
The investigational review board of Necker Children's Hospital approved this study supported by the AFIRMM (Association Française pour l'Initiative et la Recherche sur le Mastocyte et les Mastocytoses). The study was approved by the local ethics committee (Comité de Protection des personnes lle de France 6-CPP IDF 6, number 9300). All the subjects gave their written informed consent. The use of a computer database to store personal information was authorized by the French National Data Protection Commission (CNIL authorization \#1445939).

\section{Clinical evaluation and laboratory testing}

The main features of each patient were recorded on the day of the procedure (by MOC) and included mast cell activation symptoms (fatigue, pruritus, flushing, anaphylactic reactions, angioedema, diarrhea and/or gastrointestinal pains, pollakiuria (frequent urination)), clinical examination with neurological testing, B- and C-findings (that is, signs of aggressiveness) according to the validated classification criteria, serum tryptase level, past and present treatment history of mastocytosis (symptomatic as well as cytoreductive therapies) and all other drugs, notably psychoactive substances. Cardiovascular risk factors including tobacco consumption, hypertension, overweight, diabetes and cannabis use were recorded. The mental state of patients was assessed in all but one patient by a psychiatrist (RG) using the French version of the Mini International Neuropsychiatric Interview ${ }^{18}$ to diagnose psychiatric conditions according to DSM-IV-TR criteria ${ }^{18}$ and auto-questionnaires, including the Symptom Check List $90 \mathrm{R}^{19}$ and the Modified Fatigue Impact Scale. ${ }^{13}$

Laboratory tests included serum tryptase level, skin and/or bone marrow histological analysis and/or bone marrow aspirate analysis, C-KIT mutational status obtained from skin and/or bone marrow molecular analysis.

\section{Brain imaging}

All the subjects were scanned on a 1.5 T Signa General Electric scanner to obtain both morphological and perfusion sequences. The protocol included a three-dimensional T1-weighted FSPGR (Fast Spoiled Gradient echo) sequence (repetition time (TR): $16.4 \mathrm{~ms}$, echo time (TE): $7.2 \mathrm{~ms}$, bandwidth: $11.9 \mathrm{kHz}$, inversion time (TI): $550 \mathrm{~ms}$, field of view (FOV): $22 \times 22 \mathrm{~cm}^{2}$, slice thickness: $1.2 \mathrm{~mm}$, acquisition matrix: $288 \times 224$, NEX: 0.75 ) without injection, a three-dimensional T1-weighted sequence with injection of Gadolinium (TR: 7000 ms, TE: 97.2 ms, EC: 1/1, bandwidth: $35.7 \mathrm{kHz}$, FOV: $24 \times 24 \mathrm{~cm}^{2}$, slice thickness: $4.0 \mathrm{~mm}$, acquisition matrix: $320 \times 320$, NEX: 1.50), an axial T2-weighted sequence (TR: $12 \mathrm{ms,} \mathrm{TE:} 3.6 \mathrm{ms,}$ EC: $1 / 1$, bandwidth: $15.6 \mathrm{kHz}, \mathrm{Tl}: 550 \mathrm{~ms}$, FOV: $24 \times 18 \mathrm{~cm}^{2}$, slice thickness: $1.6 \mathrm{~mm}$, acquisition matrix: $320 \times 256$, NEX: 1.00 ) and an Axial FLAIR (Fluid Attenuation Inversion Recovery) sequence (TR: 9002 ms, TE: $154.3 \mathrm{~ms}$, EC: 1/1, bandwidth: $31.2 \mathrm{kHz}, \mathrm{Tl}: 2250 \mathrm{~ms}$, FOV: $22 \times 22 \mathrm{~cm}^{2}$, slice thickness: 4 mm, NEX: 1.00 , acquisition matrix: $320 \times 224$ ). ASL (arterial spin labeling) sequence was performed to measure the cerebral blood flow using pulsed continuous ASL (pseudo-continuous) in the axial scan plane with post-label delay of $1025 \mathrm{~s}$ with 40 slices of $4 \mathrm{~mm}$ (TR: $4554 \mathrm{~ms}$, TE: $10.5 \mathrm{~ms}$, EC: 1/1, TI: 1025 ms, FOV: $24 \times 24 \mathrm{~cm}^{2}$, NEX: 3.00 , acquisition matrix: $512 \times 8$ ).

The MRI images were pre-processed using Statistical Parametric Mapping (SPM8 software from the Wellcome Department of Cognitive Neurology London www.fil.ion.ucl.ac.uk/spm/software/spm8) implemented in Matlab (Mathworks, Sherborn, MA, USA) and analyzed using a voxelbased approach. Several steps were required regarding the pre-processing part of the analysis. A DICOM to NIFTI conversion was necessary before these steps. First, native $\mathrm{T} 1$ images were segmented into gray matter, white matter and cerebrospinal fluid using the segmentation tool of SPM8. Then, the gray matter resulting from that segmentation was normalized to MNI152 space using the SPM default template. Because patients may have moved between the different acquisitions causing possible geometric changes such as rotations, ASL images were coregistered to the native gray matter images. Consequently, the ASL images were normalized by applying the deformation matrices from the gray matter normalization process. The resulting images were smoothed using a $10 \mathrm{~mm}$ isotropic Gaussian filter.

\section{Visual inspection of the MRI}

The MRI scans were interpreted independently by two board-certified radiologists experienced in neuroradiology (NB, ON). Structural malformations or signal abnormalities of gray and white matter were rated for each examination. The white matter abnormalities (WMA) were classified as multiple punctate or plaque-like confluent hypersignals on T2 and FLAIR 
sequences. Multiple punctate WMA were further classified as periventricular, deep or subcortical abnormalities.

\section{Statistical analysis}

Statistical analyses of clinical data were performed using the $R$ statistics software (www.r-project.org). A detailed description of clinical features of each group is provided as a Supplementary Table; individual data are available upon request. The differences between subgroups (diagnostic subgroups or WMA versus non-WMA subgroups) were assessed through uncorrected two-tailed $t$-tests when the independent variable was continuous (Symptoms check list SCL score, age and tryptase level) and through chi-squared tests when the independent variable was categorical (depression diagnostic, presence of WMA, presence of neurological signs and migraine) or through Fisher's exact test when required (one or more expected count below 5). The assumptions of each statistical test used were checked, including equality of variances, and statistical significance was set at 0.05 . The SCL scores were analyzed with an ANOVA (analysis of variance) with $\mathrm{SCL}$ dimension as a factor, and subsequent uncorrected pairwise $t$-tests to identify specific dimensions of symptoms in patients. A specific analysis of the depression dimension was performed with a factor analysis to determine factors that best explained interindividual differences (the details of the method used are described with the results).

Voxel-based analyses were performed with SPM8 on smoothed and normalized ASL images to compare different groups within a mask encompassing all coronal slices with $y>-30 \mathrm{~mm}$.

We compared the subgroups with whole-brain analysis using an ANOVA across three subgroups (WMA, non-WMA and controls) and a t-test between depressed and non-depressed patients, with a statistical threshold set to $P<0.05$ corrected for multiple comparisons. The age covariate was considered. When an ANOVA was used, subsequent two-sided pairwise $t$-tests were performed within the mask resulting from the previous ANOVA to directly compare the different subgroups. The relationship between brain perfusion (ASL) and symptoms ( $\mathrm{SCL}$ factors arising from the factor analysis) was analyzed with Pearson's correlation analysis.

An exploratory analysis was performed to compare depressed and nondepressed patients in a region of interest, the subgenual cingulate cortex which is considered a key structure in the pathophysiology of depression (seminal reference ${ }^{20}$ for reviews see refs $21-23$ with a demonstration by Mayberg et al. ${ }^{24}$ of its causal involvement through deep brain stimulation). A $t$-test analysis was therefore performed between these subgroups on a $5 \mathrm{~mm}$ radius sphere centered on $x=-10 ; y=28 ; z=-12$, which are coordinates taken from Mayberg et al., ${ }^{24}$ with a threshold set to $P<0.05$.

\section{RESULTS}

A total of 72 subjects were enrolled in the study: 39 patients (referred to hereafter as the test cohort, mean age: $44.2 \pm 11.8$ years, age range: $15-65$ years, 6 men) and 33 healthy controls (mean age: $39.2 \pm 12.8$ years, age range: $23-60$ years, 11 men), with no significant difference between patients' age and controls' age $(P$-value $>0.1)$.

\section{Main clinical features}

In the test cohort, $12 / 39$ patients (30.8\%) had CM, 26/39 patients $(66.7 \%)$ had ISM and $1 / 39$ patient $(2.6 \%)$ had SSM. The mean length of time for the first mastocytosis-related symptom was 17 years and that of diagnosis confirmation was 13.9 years. Skin involvement was reported for all the patients in the test cohort with a vast majority of maculopapular CM skin lesions (previously denominated urticaria pigmentosa) in $92.3 \%$ of patients. Although variable in type and intensity, chronic MCA, with acute exacerbations, were identified in all patients. The most prevalent symptoms were asthenia (97.4\%), gastrointestinal disorders (94.8\%) and musculoskeletal pains, notably headaches (84.6\%), flushing (82\%), pruritus (74.3\%) and pollakiuria (59\%). The evaluation of symptoms following the grading introduced by Valent et al. ${ }^{25}$ showed that MCA symptoms in all the patients were graded 2 or 3 , with frequent mild-to-severe symptoms requiring daily therapy resulting in satisfactory (grade 2) or unsatisfactory (grade 3 ) control. None had severe adverse events requiring immediate therapy and hospitalization (grade 4). Symptomatic treatment consisted mainly of antihistamines (87\%). Approximately $41 \%$ of patients from the test cohort had received one to five previous lines of cytoreductive treatment, mainly tyrosine kinase inhibitor, due to refractory and disabling MCA. Moreover, at the time of this study, $20 / 39$ patients $(51.3 \%)$ from the test cohort were in need of a first or new line of cytoreductive therapy, stressing the burden of MCA in this cohort. Importantly, no C-finding was observed either at diagnosis or follow-up even in the SSM patient. A molecular analysis revealed expected results with a D816V C-KIT mutation in $83.3 \%$ of patients and wild-type C-KIT in the others. Finally, bone demineralization, another complication of chronic mast cell degranulation, was observed in $64 \%$ of cases.

Considering psychiatric evaluation of patients from the test cohort, $12 / 39$ patients (30.8\%) received a diagnosis of major depression, intermittent explosive disorder was diagnosed in 2/39 patients $(5.2 \%)$, with antisocial personality disorder, obsessive compulsive disorder, alcohol dependence and panic disorder each being diagnosed in $1 / 39$ patients (2.3\%). Mean scores were not identical for all dimensions of SCL90, $(F(9,380)=6.37, P<0.001)$. Three scores were significantly higher than the others (namely, somatization, obsessive compulsive and depression) with uncorrected pairwise $t$-tests showing no difference between these three dimensions and a significant difference with all other dimensions. The SCL90 depression dimension correlated with a clinical diagnosis of depression, the depression dimension being higher in the clinically depressed subgroup of patients from the test cohort $(n=13)$ compared with the non-clinically depressed subgroup ( $n=26$; $t$-test, $P=0.0367$ ). No other dimension of the SCL90 differed between these clinically depressed versus nonclinically depressed subgroups (that is, $P>0.1$ ). A factor analysis was performed on the depression dimension to determine the specific items, from the 13 items constituting the depression dimension, that contributed most to the observed interindividual differences. The Kaiser-Meyer-Olkin measure of sample adequacy was 0.86 (corresponding to very good adequacy) and Bartlett's test was significant (Chi-square $=409, P<0.001$ ). Factor analysis was then performed on three factors (a number found to be adequate using a mix of the Kaiser criterion, Variance explained and Scree test), using a promax rotation with a power of 4 . Retaining loadings above 0.7, factor 1 captured some key depressive symptoms such as feelings of loneliness and ruminations (items 29, 30 and 31), factor 2 captured elements of severity with feelings of guilt and helplessness leading to suicidal ideation (items 15, 22 and 26) and factor 3 captured a dimension of lack of motivation and energy (item 71: everything seems to be an effort). To ascertain the consistency of these dimensions, we analyzed inter-item correlation using Cronbach's alpha test for factors 1 and 2 (no correlation was necessary for factor 3 having only one item). This yielded a Cronbach's alpha of 0.92 for factor 1 and 0.89 for factor 2, which is very satisfactory. Correlation between factor 3 with either factor 1 or 2 was lower (Pearson's correlation coefficient of 0.34 and 0.39 , respectively) than the correlation between factors 1 and 2 (0.644). Factors 1 and 2 were mostly expected; however, factor 3 represented an unforeseen independent dimension that echoed patient complaints heard in clinical practice about a lack of motivation or energy. Tryptase levels were not significantly different between patients with depression and those without and did not correlate with the SCL90 factors.

\section{Neuroimaging results}

Visual inspection of MRI. MRI was rated as abnormal in 19/39 patients (49\%), and three main types of abnormalities were found: WMA $(n=16)$, plaque-like areas $(n=2)$, meningiomas $(n=2)$ and cerebellar atrophy $(n=1)$. These abnormalities occurred in combination in two patients. 


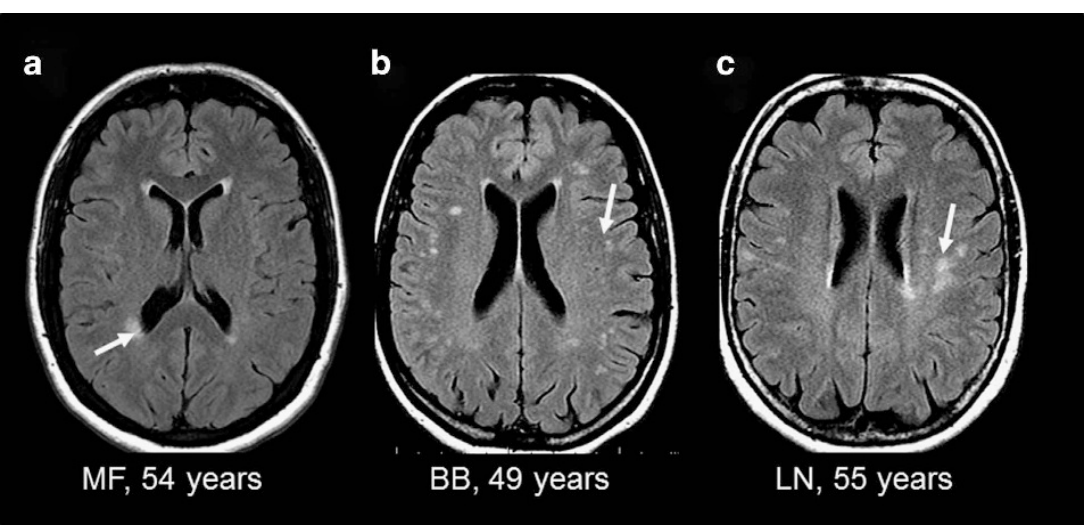

Figure 1. White matter abnormalities (WMA) in mastocytosis patients. Three mastocytosis patients illustrating the main categories of white matter signal abnormalities on axial Fluid Attenuation Inversion Recovery (FLAIR) weighted sequence: The WMA were very intense compared with the adjacent white matter on FLAIR sequences and did not involve the basal ganglia. (a) Periventricular WMA. White matter hyperintensity relatively symmetrical bilaterally at the posterior and anterior horns of the lateral ventricles. There was no deformation of the lateral ventricular contour adjacent to these lesions (see arrow). (b) Punctate subcortical T2/FLAIR hyperintensity. Abnormalities were found in the subcortical $U$ fibers of white matter (see arrow). They were asymmetric and homogeneous, and no findings suggest that necrosis was present. (c) Deep white matter. Abnormal findings were found in the deep juxta-ventricular white matter (see arrow). They were asymmetric and homogeneous.

In the group of CM patients, we found five patients with WMA and one plaque-like area and six normal MRIs. The WMA involved the periventricular white matter fibers $(n=2 / 5)$, the deep juxtaventricular white matter $(n=3 / 5)$ and the subcortical $U$ fibers $(n=5 / 5)$. In the group of ISM/SSM patients, we found 11 patients with WMA, 1 plaque-like area, 2 meningiomas and 16 normal MRI. The WMA involved the periventricular white matter fibers $(n=6$ /11), the deep juxta-ventricular white matter $(n=7 / 11)$ and/or the subcortical $U$ fibers $(n=8 / 11)$.

The WMA were small ( $<2 \mathrm{~mm}$ ), asymmetric and homogeneous, and they were very intense compared with the adjacent white matter on T2 and FLAIR sequences. No findings suggested that necrosis was present. Their localizations showed inter-patient variability. They could involve the periventricular white matter fibers $(n=8 / 16)$, the deep juxta-ventricular white matter $(n=8 / 16)$ and/or the subcortical $U$ fibers $(n=13 / 16$; Figure 1$)$. The WMA were equally distributed in the CM and ISM/SSM groups (5 patients among 12 CM and 11 patients among 27 ISM/SSM, respectively, $P=1$, two-sided Fisher's exact test of independence). The overall frequency of neurological complaints was comparable in the CM and ISM/SSM groups (24 and 34, respectively, Chi-square $P=0.40$ ). Neurological complaints frequency was also comparable by type of symptom: migraine (9/12 and $16 / 27$, Chi-square $P=0.87)$, symptoms suggesting peripheral neuropathy $(6 / 12$ and $10 / 27$, Chi-square $P=0.87)$, dizziness $(6 / 12$ and $5 / 27$, Fisher's $P=0.17)$, numbness of one or more limbs (1/12 and $1 / 27$, Fisher's $P=0.54)$, movement control complaints suggesting dyskinesia $(0 / 12$ and $2 / 27$, Fisher's $P=1)$, speech disorder $(1 / 12$ and $0 / 27$, Fisher's $P=32)$ and visual complaints $(1 / 12$ and $0 / 27$, Fisher's $P=0.32)$. The $S C L$ depression subscale was lower in the CM than in the ISM/SSM group (1.04 versus 1.77 raw score, $t$-test $P=0.035$ ), but the total SCL score was not different ( 0.86 versus 1.32 raw score, $t$-test $P=0.12$ ) and the frequency of depression diagnostic was not significantly different (2/12 and 10/27, Fisher's $P=0.27)$.

Plaques-like areas with hyperintensity on T2 and FLAIR were present in two patients. One patient had two plaque-like areas: one plaque-like area was localized in the right temporal pole/ amygdalian region and the hyperintensity on FLAIR decreased over 2 months (Figure 2a); the second plaque-like area was localized in the right periventricular region with a contrast
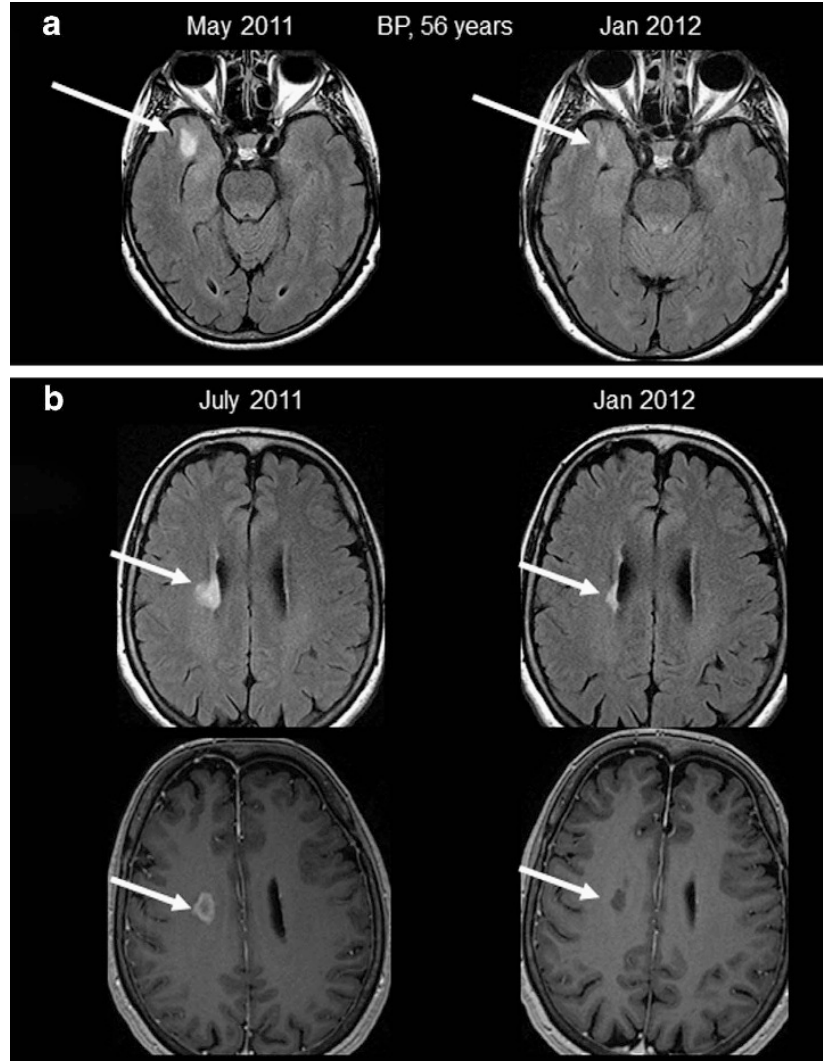

Figure 2. Plaque-like areas. (a) A 56-year-old mastocytosis patient had two plaque-like areas on magnetic resonance imaging (MRI): one plaque-like area was localized in the right temporal pole/ amygdalian region. The axial Fluid Attenuation Inversion Recovery (FLAIR) sequence showed important hyperintensity without contrast enhancement, which decreased in 7 months. (b) The second plaquelike area was localized in the right periventricular region as shown by the axial FLAIR sequence (up) with an important contrast enhancement (axial T1 with injection on bottom row) that also decreased at 6 months. 


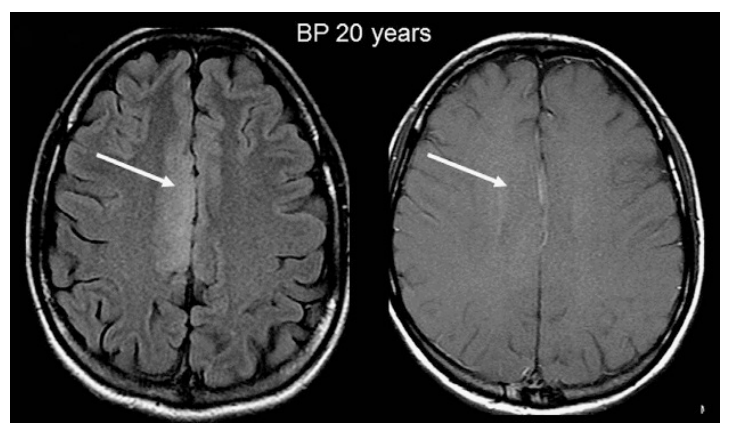

Figure 3. Plaque-like area mimicking a tumor. A 20-year-old mastocytosis patient had a plaque-like area that was localized in the right frontal superior cortex. The axial Fluid Attenuation Inversion Recovery (FLAIR; left) and axial contrast T1 FSE weighted images (right) showed an important hyperintensity without any mass effect or contrast enhancement mimicking a low-grade oligodendroglioma (arrows).

enhancement that disappeared over 6 months (Figure 2b). For the second patient, one plaque-like area was localized in the frontal superior lobe and was mimicking a low-grade oligodendroglioma without any mass effect or contrast enhancement (Figure 3).

When looking for clinical features that could explain these morphological brain abnormalities, the comparison of subgroups with or without WMA ( $n=16$ and 23 , respectively) revealed no significant differences: the mean age in the WMA subgroup was 49 years (range 15-65) versus 40.8 years (range $21-57$ ) in the subgroup without WMA; the sex ratio was $4 / 12$ versus $3 / 20$, respectively, largely in favor of an over-representation of females in both groups; there was a similar past history of mastocytosis with the mean time from diagnosis being 17 years (range 3-35) versus 15.4 years (range 5-33), respectively; and no definite conclusion can be drawn concerning the impact of previous exposure to cytoreductive drugs, but the cytoreductive therapeutic profile appeared relatively similar to the two subgroups with $25 \%$ of patients receiving one to five treatment lines (including alpha-interferon, cladribine, tyrosine kinase inhibitor, thalidomide and rapamycin) versus $34 \%$ of patients receiving one to three treatment lines (including alpha-interferon, cladribine, tyrosine kinase inhibitor). Tryptase levels were similar in both groups $(t=0.62, P=0.54)$. Headache and/or migraine incidence did not significantly differ between the groups (Fisher's exact test, $P=0.50$ and $P=0.53$, respectively). Crucially, cardiovascular risk factors were equally present in patients with or without WMA, respectively: tobacco (in $4 / 16$ patients, $25 \%$ versus $8 / 23$ patients, $34 \%$, Fisher's exact test $P=0.74$ ); hypertension (in $2 / 16$ patients, $12.5 \%$ versus $2 / 23$ patients, $8.7 \%, P=1$ ); overweight (in $3 / 16$ patients, $18.7 \%$ versus $4 / 23$ patients, $17.4 \%, P=1$ ); chronic cannabis abuse (in $1 / 16$ patient, $6.3 \%$ versus $2 / 23$ patients, $8.7 \%$, $P=1$ ); and diabetes (in $1 / 16$ patient, $6.3 \%$ versus $0 / 23$ patient, $0 \%$, $P=0.42$ ). Three representative patients with brain pathological abnormalities (WMA and/or plaque-like lesions) were extensively assessed to eliminate other diagnoses than mastocytosis (see Table 1). In all three cases, an inflammatory (notably multiple sclerosis), infectious or tumoral disease, as well as a cardiovascular etiology (leukoaraiosis), could be stringently eliminated. Regarding psychiatric evaluation, there was no significant difference in the occurrence of depression among patients with or without WMA; specifically $4 / 16(25 \%)$ versus $8 / 23(34.8 \%)$, respectively (Fisher's exact test, $P=0.73$ ).

Voxel-based analysis. The ANOVA analysis on all three groups (WMA patients, non-WMA patients and healthy control subjects) showed a significant difference in cerebral blood flow measured in the left putamen $(-18,12,-1 ; z$-score $4.54,620$ voxels, $P<0.001$ uncorrected, $P=0.029$ corrected, Figure $4 a)$. There were also right putamen changes, with a less significant effect that did not survive our whole-brain threshold $(22,18,-15 ; z=3.51,140$ voxels, $P<0.001$ uncorrected, $P=0.678$ corrected). In the between-group analyses (Figure 4b), WMA mastocytosis patients showed a significant hyperperfusion in the left putamen compared with both healthy control subjects and non-WMA mastocytosis patients $\left(P<0.001\right.$ uncorrected, $P=2.3 \times 10^{-5}$ corrected, $z=5.01$ and $P<0.001$ uncorrected, $P=0.0013$ corrected, $z=4.05$, respectively). At a more lenient threshold, WMA mastocytosis patients also had an increase of cerebral blood flow in the right putamen compared with both non-WMA mastocytosis patients and control subjects $\left(P=0.002\right.$ uncorrected, $Z=2.93$ and $P=2.1 \times 10^{-5}$ uncorrected, $z=4.08$, respectively). Non-WMA mastocytosis patients did not differ from controls in the left or right putamen $(P=0.13$ uncorrected, $z=1.09$ and $P=0.07$ uncorrected, $z=1.48$, respectively).

Finally, we tested whether brain activity correlated with clinical scores using the factors found to best explain interindividual differences in depression scores, factors 1 through 3 of the factor analysis. Putamen perfusion correlated strongly with the lack of motivation dimension (factor 3, item 71, Pearson's correlation $r=-0.60, P<0.001$, Figure 4c). There was no correlation with the main symptoms dimension (factor $1, r=-0.31, P=0.06$ ) and a marginal correlation with the severity dimension (factor 2, $r=-0.08, P=0.63$ ).

Based on the depression diagnosis on a small subset of patients, we compared cerebral blood flow in depressed versus nondepressed mastocytosis patients but found no difference in cerebral blood flow in a whole-brain analysis $(P>0.1)$. For exploratory purposes, mean ASL perfusion was calculated within the subgenual cingulate cortex defined a priori following previous publications in depression, as detailed in the 'Materials and methods' section. The region of interest was a $5 \mathrm{~mm}$ radius sphere centered on $x=-10 ; y=28 ; z=-12$. This analysis yielded a significant decrease in cerebral blood flow (hypoperfusion) in the subgenual cingulate cortex in depressed compared with nondepressed mastocytosis patients $(P=0.002$ uncorrected, $P=0.012$ corrected, $T=3.06$ and $z=2.96$, Figures $5 \mathrm{a}$ and b). Again, perfusion in this region correlated strongly with the lack of motivation dimension of the depression subscale of the SCL90 (factor 3, item 71 , Pearson's correlation $r=-0.460, P=0.003$, Figure $5 c$ ).

\section{DISCUSSION}

The aim of this study was to determine the neural basis of psychiatric and cognitive complaints in indolent forms of mastocytosis through systematic MRI and psychiatric evaluations. In the test cohort of 39 mastocytosis patients with a neuropsychiatric complaint, we found that (i) $49 \%$ of patients had morphological brain abnormalities, mainly abnormal punctuated white matter hypersignals (that is, WMA); (ii) an increased perfusion was demonstrated in the putamen in mastocytosis patients compared with control subjects, an increase that was demonstrated to be specific to those mastocytosis patients with WMA; (iii) putamen perfusion was negatively correlated with the motivation dimension of the depression subscore of the SCL90; and (iv) an exploratory analysis showed a decreased perfusion in the subgenual cingulate cortex in depressed compared with nondepressed mastocytosis patients.

Among the test cohort of mastocytosis patients with psychocognitive complaints $(n=39), 41 \%$ had punctuated WMA. The pathological character for isolated periventricular hypersignals could be confounded by age because such signals are seen in patients over the age of 40 years with leukoariosis. However, only $1 / 16$ patients $(6.3 \%)$ from the WMA subgroup with isolated periventricular hypersignals was over 40 years old. Furthermore, 
Table 1. Neurologic expert analysis of three patients with WMA and/or plaque-like lesions

\begin{tabular}{|c|c|c|c|}
\hline & $F, 53$ years & $F, 57$ years & $F, 21$ years \\
\hline Subjective symptoms & $\begin{array}{l}\text { Rare headaches } \\
\text { Rare paresthesia }\end{array}$ & $\begin{array}{l}\text { Usual migraine } \\
\text { Some dysesthesia }\end{array}$ & $\begin{array}{l}\text { Headaches } \\
\text { Cognitive complaints } \\
\text { Paresthesia }\end{array}$ \\
\hline Brain MRI results & WMA & $\begin{array}{l}\text { Two plaque-like areas and WMA } \\
\text { Figures } 2 a \text { and } b \text {, }\end{array}$ & $\begin{array}{l}\text { One plaque-like area } \\
\text { Figure } 3\end{array}$ \\
\hline $\begin{array}{l}\text { Cardiovascular risk } \\
\text { factors }^{\text {a }}\end{array}$ & None & None & None \\
\hline $\begin{array}{l}\text { Neurological } \\
\text { examination }^{\mathrm{b}}\end{array}$ & Normal & Normal & Normal \\
\hline $\begin{array}{l}\text { Cardiovascular } \\
\text { expertise }\end{array}$ & $\begin{array}{l}\text { ECG } \\
\text { TTE } \\
24 \text { h holter AP } \\
\text { Effort test }\end{array}$ & $\begin{array}{l}\text { ECG } \\
\text { TTE }\end{array}$ & $\begin{array}{l}\text { ECG } \\
\text { TTE }\end{array}$ \\
\hline Blood tests ${ }^{c}$ & $\begin{array}{l}\text { Normal except a slight } \\
\text { cholesterol excess } \\
\text { resolved by diet }\end{array}$ & $\begin{array}{l}\text { Normal except the same slight oligoclonal } \\
\text { aspect as in CSF }\end{array}$ & Normal \\
\hline $\begin{array}{l}\text { Lumbar puncture } \\
\text { Proteinorachy } \\
\text { Glycorachy } \\
\text { LDH level } \\
\text { Electrophoresis and } \\
\text { immune-EP } \\
\text { Cytology } \\
\text { Microbial cultures } \\
\text { Other }\end{array}$ & $\begin{array}{l}\text { All normal notably } \\
\text { Proteinorachy } \\
\text { Cytology: no cells }\end{array}$ & $\begin{array}{l}\text { All normal except proteinorachy } 0.72 \mathrm{~g} \mathrm{I}^{-1} \\
\text { then } 0.68 \mathrm{~g} \mathrm{I}^{-1} \text { with slight lgG oligoclonality } \\
\text { as in the serum invalidating an intrathecal } \\
\text { synthesis and no cells }\end{array}$ & $\begin{array}{l}\text { All normal notably } \\
\text { Proteinorachy } \\
\text { Cytology: no cells } \\
\text { Complemented by } \\
\text { Tryptase dosage: undetectable } \\
\text { FC: no mast cells }\end{array}$ \\
\hline EEG & Normal & Not done & $\begin{array}{l}\text { Rare multifocal theta spindles without } \\
\text { paroxystic aspect }\end{array}$ \\
\hline Evocate potentials & Normal & Not done & Not done \\
\hline $\begin{array}{l}\text { Magnetic resonance } \\
\text { spectroscopy }\end{array}$ & Not done & No inflammatory or tumoral aspect & $\begin{array}{l}\text { Choline increase and NAA peak decrease, } \\
\text { without hypervascularization }\end{array}$ \\
\hline Brain lesion biopsy & Not done & Not done & $\begin{array}{l}\text { Stereotaxic biopsy in favor of a glioma but } \\
\text { without certainty (rare glial cells expressing } \\
\text { IDH-1R132H). No pathological mast cell } \\
\text { infiltrate. }\end{array}$ \\
\hline
\end{tabular}

Abbreviations: AP, arterial pressure; CSF, cerebrospinal fluid; ECG, electrocardiogram; EEG, electroencephalogram; EP, electrophoresis; $F$, female; FC, flow cytometry using anti-CD117, anti-CD25, anti-CD2 immuno-markers; IgG, immunoglobulin G; MRI, magnetic resonance imaging; NAA, N-acetyl-aspartate; TTE, transthoracic echocardiography; WMA, white matter abnormality. ${ }^{\text {} H y p e r t e n s i o n, ~ d i a b e t e s, ~ d y s l i p i d e m i a, ~ s m o k i n g ~ a n d ~ o t h e r ~ d r u g ~ a b u s e, ~ a n d ~ p a s t ~ h i s t o r y ~ o f ~}$ thrombosis. ${ }^{b}$ Performed by an expert neurologist. ' A complete blood analysis was performed notably inflammatory markers (C-reactive protein, fibrinogen), hemostasis tests, lipid and diabetes tests, thyroid function, serum electrophoresis and immune-fixation, HIV serology.

the WMA and non-WMA subgroups were well-matched for age and cardiovascular risk factors. Moreover, in $14 / 16$ patients ( $87.5 \%)$ these periventricular hypersignals were not isolated but associated with deep white matter hypersignals (4/16) or subcortical hypersignals (5/16). Of the 16 WMA patients, 13 also presented with subcortical white matter hypersignals, and these types and topography of anomalies could be seen in vasculitis diseases. Among the 16 patients, 7 presented with an association of abnormal deep and subcortical white matter hypersignals, 8 presented with deep white matter hypersignals similar to what is seen in inflammatory disease such as multiple sclerosis. These morphological abnormalities were not related to any features of mastocytosis such as the sex ratio, mean duration of illness or history of treatments. An inflammatory (notably multiple sclerosis), infectious or tumoral disease could be stringently eliminated through extensive explorations in three patients.

In two of the three extensively explored patients, we also found hyperintensity 'plaque-like areas' on FLAIR sequences. One plaque-like area was localized in the frontal superior lobe and was mimicking a low-grade oligodendroglioma. A biopsy of the lesion was not very contributive as it was not possible to distinguish between oligodendroglioma tumor and inflammatory disease. The fact that no definite histological diagnosis was done in this patient, including the absence of mast cell infiltrate, does not exclude the role of mastocytosis in this lesion. Indeed, the recruitment and proliferation of inflammatory and glial cells could be favored by mast cell neuro-mediators. Similarly, psychocognitive symptoms, as well as the neuroimaging abnormalities, may result from the impact of mast cell mediators rather than mast cell infiltrates. Similar brain abnormalities might be found in patients with neuropsychiatric impairments who are suffering from MCA syndrome in which neither pathological mast cell infiltration nor proliferation is detected. In these cases, symptoms depend exclusively on aberrant mast cell mediator release. Systematic brain imaging in those patients would thus be of interest and even used as diagnostic criteria of MCA. In the other patients, the plaque-like lesion was localized in the right periventricular region with a contrast enhancement and it disappeared over a period of 6 months. This same patient had another plaque-like abnormality in the right amygdala. Of most 
a

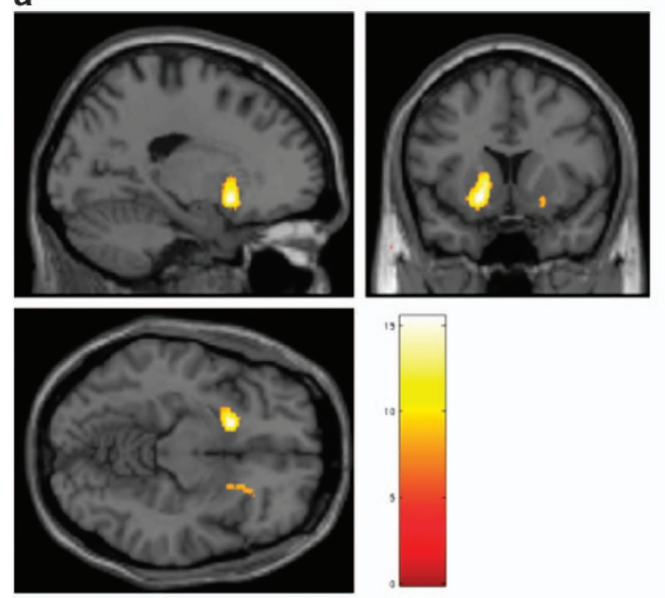

b

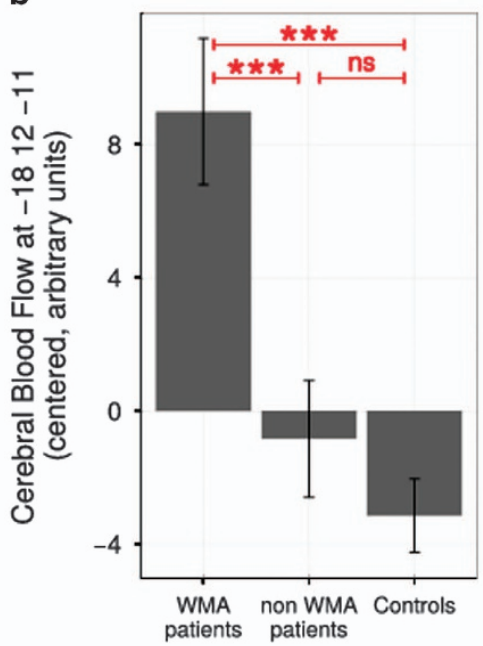

C

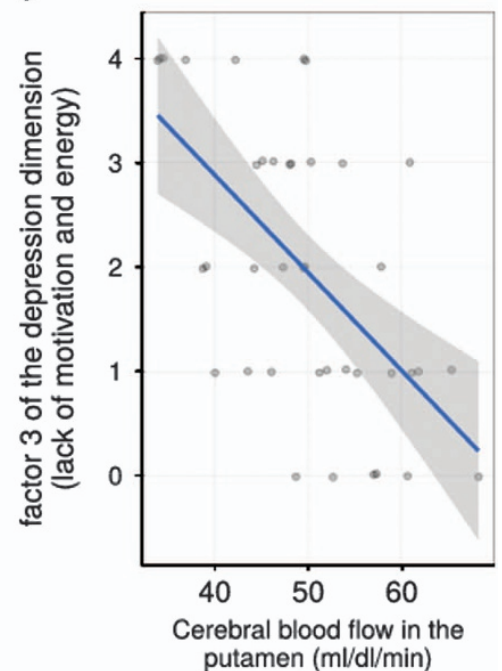

Figure 4. (a) Hyperperfusion in bilateral basal ganglia in mastocytosis patients with white matter abnormalities (WMA): analysis of variance (ANOVA) test for the three groups (WMA patients, non-WMA patients and controls) at the level $P=0.001$ uncorrected, peak voxel in the left putamen $(x=-18, y=12, z=-11), P=0.03$ corrected. (b) Cerebral blood flow measured with MRI/ASL (arbitrary units) in the left putamen $(x=-18, y=12, z=-11){ }^{* * *} \mathrm{p}<0.001$. WMA patients had a significant increase in cerebral blood flow in this region compared with non-WMA patients $(P<0.001)$ and to control subjects $(P<0.001)$. Non-WMA patients did not differ from controls. Error bars represent standard error of the mean (s.e.m.). (c) Significant correlation between left putamen perfusion and factors 3, that is, motivation, arising from the factor analysis of SCL90 depression dimension. The shaded area represents the $95 \% \mathrm{Cl}$. ASL, arterial spin labeling; Cl, confidence interval; MRI, magnetic resonance imaging; NS, not significant.

a

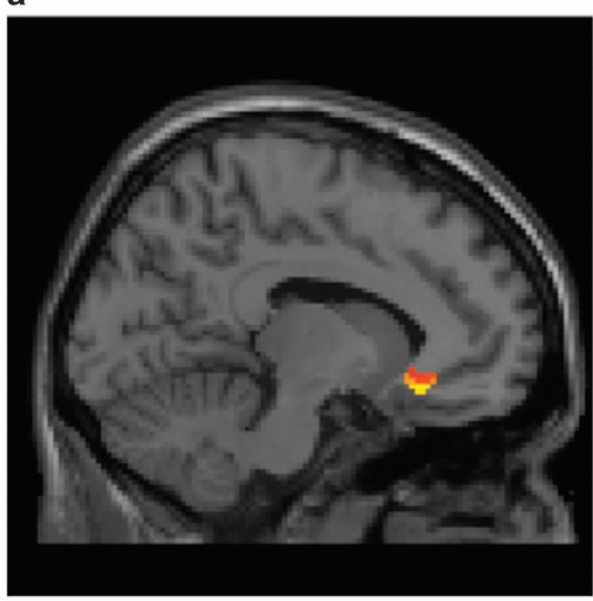

b

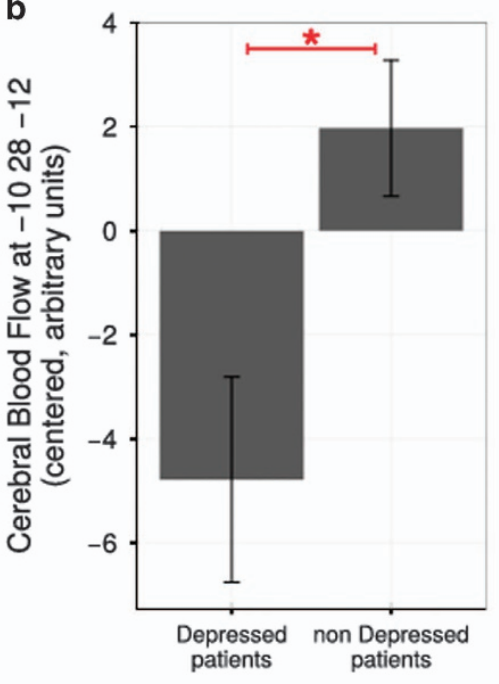

C

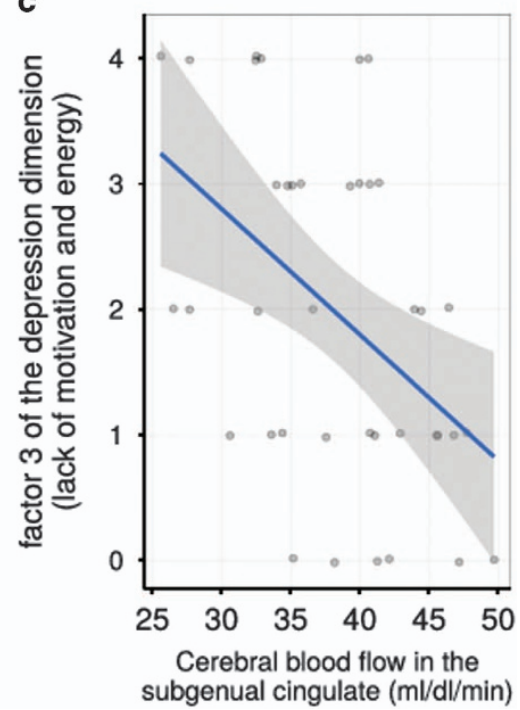

Figure 5. (a) Hypoperfusion in the anterior cingulate cortex in mastocytosis patients with depression compared with patients without depression in an ROI analysis: $t$-test between depressed and non-depressed patients at the level $P=0.05$ uncorrected, peak voxel in the anterior cingulate cortex $(x=-10, y=28 ; z=-12), P=0.012$ corrected. (b) Cerebral blood flow measured with MRI/ASL (arbitrary units) in the subgenual cingulate cortex $(x=-10, y=28 ; z=-12)$. ${ }^{*} p<0.05$. Depressed patients had a significant decrease in cerebral blood in this region compared with non-depressed patients ( $t$-test, $P=0.012$ corrected). Error bars represent standard error of the mean (s.e.m.). (c) Significant correlation between subgenual cingulate perfusion and factors 3 arising from the factor analysis of SCL90 depression dimension. The shaded area represents the $95 \% \mathrm{Cl}$. ASL, arterial spin labeling; $\mathrm{Cl}$, confidence interval; MRI, magnetic resonance imaging; ROI, region of interest.

interest, this patient received a diagnosis of panic disorder, a condition that resolved with antidepressant treatment (escitalopram $10 \mathrm{mg}$ per day) and coincided with the disappearance of the right amygdalian lesion. This pattern is consistent with the potential involvement of amygdala dysfunction in panic disorder pathophysiology, ${ }^{26,27}$ with mast cells promoting anxiety ${ }^{28}$ through a direct anatomical relationship.
Increased perfusion in the putamen was specific to WMA mastocytosis patients. Both the WMA and putamen hyperperfusion might be related to a common mechanism such as inflammation and/or ischemia through mast cell activation. ${ }^{29}$ WMA might also disrupt the prefrontal and basal ganglia networks, ${ }^{30}$ linking cortical impairment in the prefrontal cortex by WMA and increased perfusion in the basal ganglia. In another 
dysconnectivity hypothesis, WMA might impair cortico-cortical connectivity, including long-range connectivity, resulting in working memory and attention impairment ${ }^{31,32}$ and eventually in cognitive efforts that basal ganglia perfusion would translate. ${ }^{33,34}$ Such a cortico-cortical impairment would be reminiscent of multiple sclerosis, ${ }^{35}$ in which cognitive symptoms and fatigue have a huge burden. ${ }^{36-38}$ We might also draw a link with another condition with high prevalence of fatigue, systemic lupus erythematosus, in which increased basal ganglia metabolism has been recently demonstrated in a cognitive paradigm, ${ }^{39}$ as well as with a similar pattern demonstrated in patients treated with interferon alpha. ${ }^{40,41}$ However, in contrast with observations in interferon alpha-treated patients, we found a negative correlation between putamen hyperperfusion and fatigue, as approximated by the motivation factor from the SCL90 depression dimension. This discrepancy might be related to chronic brain inflammation in mastocytosis patients, in contrast with early observations after only 4 to 6 weeks of interferon alpha treatment. ${ }^{40,41}$ If putamen activation in an inflammatory context initially correlates with fatigue, a persistent activation with chronic inflammation might conversely allow compensating the sickness behavior related to inflammation.

Consistent with the inflammation hypothesis, we have recently shown that in mastocytosis, tryptophane catabolism is disturbed. ${ }^{42}$ Indeed, patients display a low level of tryptophane and serotonin associated with a higher IDO1 activity resulting in higher kynurenic and mostly quinolinic acid levels. These alterations correlate with perceived stress and depression, demonstrating mast cell involvement in inflammation pathways associated with depression. It remains to be determined whether or not these metabolic changes are correlated and/or responsible for the MRI abnormalities.

Although exploratory, the region of interest analysis hints at decreased perfusion in the sgACC of depressed versus nondepressed mastocytosis patients, consistent with the key role attributed to the sgACC in the pathophysiology of depression in the literature (for reviews, see refs 21-23; and for a causal role demonstration through deep brain stimulation, see ref. 24). However, it is noteworthy that despite early demonstrations of decreased activity or perfusion, as in our results, most neuroimaging studies demonstrated an increased activity. This result might be related to the clinical characteristics of depression in mastocytosis patients that further studies with a larger sample could determine. Reduced cingulate activity following infarcts or surgery is known to contribute to behavioral disorders including akinetic mutism, diminished self-awareness and depression, motor neglect and impaired motor initiation. ${ }^{23}$ Indeed, the strong negative correlation between sgACC perfusion and the motivation factor from the SCL90 depression dimension (everything seems to be an effort) argue for a relation between sgACC hypoperfusion and lack of motivation or energy, consistent with the crucial involvement of the cingulate cortex in motivating and 'energizing' behavior. $^{43}$

In conclusion, we found certain morphological and functional brain abnormalities to be associated with mastocytosis. It remains to be elucidated whether and how mastocytosis causes these abnormalities and whether these abnormalities are specific to mastocytosis patients featuring these neuropsychiatric symptoms. Further studies with larger groups of patients are warranted to confirm these results, along with therapeutic studies to evaluate the correlation between clinical/biological and morphological/ perfusion brain changes.

\section{CONFLICT OF INTEREST}

RG has received compensation as a member of the scientific advisory board of Janssen, Lundbeck, Roche and Takeda. He has served as a consultant and/or speaker for Astra Zeneca, Pierre Fabre, Lilly, Otsuka, SANOFI, Servier and received compensation, and he has received research support from Servier. $\mathrm{OH}$ received research funding and honorarium from $A B$ Science, and research grant from Novartis, Celgene, Hybrigenics, Inatherys Bristol Myer Squibb and Takeda. The remaining authors declare no conflict of interest.

\section{REFERENCES}

1 Carter MC, Metcalfe DD, Komarow HD. Mastocytosis. Immunol Allergy Clin North Am 2014; 34: 181-196.

2 Akin C, Valent P. Diagnostic criteria and classification of mastocytosis in 2014. Immunol Allergy Clin North Am 2014; 34: 207-218.

3 Longley BJ, Tyrrell L, Lu SZ, Ma YS, Langley K, Ding TG et al. Somatic c-KIT activating mutation in urticaria pigmentosa and aggressive mastocytosis: establishment of clonality in a human mast cell neoplasm. Nat Genet 1996; 12: 312-314.

4 Valent P, Horny HP, Escribano L, Longley BJ, Li CY, Schwartz LB et al. Diagnostic criteria and classification of mastocytosis: a consensus proposal. Leuk Res 2001; 25: 603-625.

5 Horny AC, Metcalfe DD, Swerdlow S, Campo E, Lee Harris N et al. Mastocytosis. WHO Classification of Tumours of Haematopoietic and Lymphoid Tissues, 2008, pp 53-63.

6 Arber DA, Orazi A, Hasserjian R, Thiele J, Borowitz MJ, Le Beau MM et al. The 2016 revision to the World Health Organization classification of myeloid neoplasms and acute leukemia. Blood 2016; 127: 2391-2405.

7 Valent P, Escribano L, Broesby-Olsen S, Hartmann K, Grattan C, Brockow K et al. Proposed diagnostic algorithm for patients with suspected mastocytosis: a proposal of the European Competence Network on Mastocytosis. Allergy 2014; 69: 1267-1274.

8 Hermine O, Lortholary O, Leventhal PS, Catteau A, Soppelsa F, Baude C et al. Casecontrol cohort study of patients' perceptions of disability in mastocytosis. PLoS ONE 2008; 3: e2266.

9 Rogers MP, Bloomingdale K, Murawski BJ, Soter NA, Reich P, Austen KF. Mixed organic brain syndrome as a manifestation of systemic mastocytosis. Psychosom Med 1986; 48: 437-447.

10 Smith JH, Butterfield JH, Pardanani A, DeLuca GC, Cutrer FM. Neurologic symptoms and diagnosis in adults with mast cell disease. Clin Neurol Neurosurg 2011; 113: $570-574$.

11 Moura DS, Sultan S, Georgin-Lavialle S, Barete S, Lortholary O, Gaillard R et al. Evidence for cognitive impairment in mastocytosis: prevalence, features and correlations to depression. PLOS ONE 2012; 7: e39468.

12 Moura DS, Sultan S, Georgin-Lavialle S, Pillet N, Montestruc F, Gineste P et al. Depression in patients with mastocytosis: prevalence, features and effects of masitinib therapy. PLoS ONE 2011; 6: e26375.

13 Multiple Sclerosis Council for Clinical Practice Guidelines. Fatigue and Multiple Sclerosis: Evidence-Based Management Strategies for Fatigue in Multiple Sclerosis. Paralyzed Veterans of America: Washington, DC, USA, 1998.

14 Lauvsnes MB, Beyer MK, Appenzeller S, Greve OJ, Harboe E, Gøransson LG et al. Loss of cerebral white matter in primary Sjögren's syndrome: a controlled volumetric magnetic resonance imaging study. Eur J Neurol 2014; 21: 1324-1329.

15 Kikuchi H, Takayama M, Hirohata S. Quantitative analysis of brainstem atrophy on magnetic resonance imaging in chronic progressive neuro-Behçet's disease. J Neurol Sci 2014; 337: 80-85.

16 Mikdashi JA. Altered functional neuronal activity in neuropsychiatric lupus: a systematic review of the fMRI investigations. Semin Arthritis Rheum 2016; 45: 455-462.

17 Mak A, Cheung MW, Chiew HJ, Liu Y, Ho RC. Global trend of survival and damage of systemic lupus erythematosus: meta-analysis and meta-regression of observational studies from the 1950s to 2000s. Semin Arthritis Rheum 2012; 41: 830-839.

18 Sheehan DV, Lecrubier Y, Sheehan KH, Amorim P, Janavs J, Weiller E et al. The Mini-International Neuropsychiatric Interview (M.I.N.I.): the development and validation of a structured diagnostic psychiatric interview for DSM-IV and ICD-10. J Clin Psychiatry 1998; 59(Suppl 20): 22-33.

19 Derogatis LR. SCL-90-R: Administration, Scoring and Procedures Manual for rhe $R$ (evised) Version: Baltimore. John Hopkins University School Medicine: Baltimore, MD, USA, 1977.

20 Drevets WC, Price JL, Simpson JR Jr., Todd RD, Reich T, Vannier M et al. Subgenual prefrontal cortex abnormalities in mood disorders. Nature 1997; 386: 824-827.

21 Drevets WC, Savitz J, Trimble M. The subgenual anterior cingulate cortex in mood disorders. CNS Spectr 2008; 13: 663-681.

22 Hamani C, Mayberg H, Stone S, Laxton A, Haber S, Lozano AM. The subcallosal cingulate gyrus in the context of major depression. Biol Psychiatry 2011; 69: 301-308.

23 Sacher J, Neumann J, Funfstuck T, Soliman A, Villringer A, Schroeter ML. Mapping the depressed brain: a meta-analysis of structural and functional alterations in major depressive disorder. J Affect Disord 2012; 140: 142-148. 
24 Mayberg HS, Lozano AM, Voon V, McNeely HE, Seminowicz D, Hamani C et al. Deep brain stimulation for treatment-resistant depression. Neuron 2005; 45: 651-660.

25 Valent P, Akin C, Escribano L, Födinger M, Hartmann K, Brockow K et al. Standards and standardization in mastocytosis: consensus statements on diagnostics, treatment recommendations and response criteria. Eur J Clin Invest 2007; 37: 435-453.

$26 \mathrm{Kim}$ JE, Dager SR, Lyoo IK. The role of the amygdala in the pathophysiology of panic disorder: evidence from neuroimaging studies. Biol Mood Anxiety Disord 2012; 2: 20.

27 Dresler T, Guhn A, Tupak SV, Ehlis AC, Herrmann MJ, Fallgatter AJ et al. Revise the revised? New dimensions of the neuroanatomical hypothesis of panic disorder. J Neural Transm (Vienna) 2013; 120: 3-29.

28 Nautiyal KM, Ribeiro AC, Pfaff DW, Silver R. Brain mast cells link the immune system to anxiety-like behavior. Proc Natl Acad Sci USA 2008; 105: 18053-18057.

29 Silver R, Curley JP. Mast cells on the mind: new insights and opportunities. Trends Neurosci 2013; 36: 513-521.

30 Alexander GE, DeLong MR, Strick PL. Parallel organization of functionally segregated circuits linking basal ganglia and cortex. Annu Rev Neurosci 1986; 9: 357-381.

31 Dehaene S, Changeux JP. Experimental and theoretical approaches to conscious processing. Neuron 2011; 70: 200-227.

32 Gaillard R, Dehaene S, Adam C, Clémenceau S, Baulac M, Cohen L et al. Converging intra-cranial markers of conscious access. PLoS Biol 2009; 7: e61.

33 Schmidt L, Lebreton M, Clery-Melin ML, Daunizeau J, Pessiglione M. Neural mechanisms underlying motivation of mental versus physical effort. PLoS Biol 2012; 10: e1001266.

34 Kurniawan IT, Guitart-Masip M, Dolan RJ. Dopamine and effort-based decision making. Front Neurosci 2011; 5: 81.

35 Reuter F, Del Cul A, Malikova I, Naccache L, Confort-Gouny S, Cohen L et al. White matter damage impairs access to consciousness in multiple sclerosis. Neuroimage 2009; 44: 590-599.
36 Induruwa I, Constantinescu CS, Gran B. Fatigue in multiple sclerosis - a brief review. J Neurol Sci 2012; 323: 9-15.

37 Braley TJ, Chervin RD. Fatigue in multiple sclerosis: mechanisms, evaluation, and treatment. Sleep 2010; 33: 1061-1067.

38 Greim B, Benecke R, Zettl UK. Qualitative and quantitative assessment of fatigue in multiple sclerosis (MS). J Neurol 2007; 254(Suppl 2): ॥58-II64.

39 Mak A, Ren T, Fu EH, Cheak AA, Ho RC. A prospective functional MRI study for executive function in patients with systemic lupus erythematosus without neuropsychiatric symptoms. Semin Arthritis Rheum 2012; 41: 849-858.

40 Capuron L, Pagnoni G, Demetrashvili MF, Lawson DH, Fornwalt FB, Woolwine B et al. Basal ganglia hypermetabolism and symptoms of fatigue during interferonalpha therapy. Neuropsychopharmacology 2007; 32: 2384-2392.

41 Capuron L, Pagnoni G, Drake DF, Woolwine BJ, Spivey JR, Crowe RJ et al. Dopaminergic mechanisms of reduced basal ganglia responses to hedonic reward during interferon alfa administration. Arch Gen Psychiatry 2012; 69: 1044-1053.

42 Georgin-Lavialle S, Moura DS, Salvador A, Chauvet-Gelinier JC, Launay JM, Damaj $\mathrm{G}$ et al. Mast cells' involvement in inflammation pathways linked to depression: evidence in mastocytosis. Mol Psychiatry 2016; 21: 1511-1516.

43 Holroyd CB, Yeung N. Motivation of extended behaviors by anterior cingulate cortex. Trends Cogn Sci 2012; 16: 122-128.

(c) (1) This work is licensed under a Creative Commons AttributionNonCommercial-ShareAlike 4.0 International License. The images or other third party material in this article are included in the article's Creative Commons license, unless indicated otherwise in the credit line; if the material is not included under the Creative Commons license, users will need to obtain permission from the license holder to reproduce the material. To view a copy of this license, visit http:// creativecommons.org/licenses/by-nc-sa/4.0/

(c) The Author(s) 2017

Supplementary Information accompanies this paper on the Translational Psychiatry website (http://www.nature.com/tp) 